\title{
Selective adsorption of alkyl thiols on gold in different geometries
}

\author{
Philipp Schapotschnikow, René Pool, Thijs J.H. Vlugt* \\ Condensed Matter and Interfaces, Utrecht University, P.O. Box 80000, 3508 TA Utrecht, The Netherlands
}

Available online 22 February 2007

\begin{abstract}
The adsorption of mixtures of alkyl thiol surfactants on the Au(111) surface as well as on icosahedral gold nanocrystals (NCs) was investigated by molecular simulation. We compared the molfraction of each surfactant type on the gold structure with the molfraction of each surfactant type in the surrounding bulk solvent. For alkyl thiol surfactants with 1 to 4 carbon atoms difference, we found that, in contrast to the (111) surface, the adsorption selectivity on a $\mathrm{NC}$ is almost identical to that in the solvent.
\end{abstract}

(c) 2007 Elsevier B.V. All rights reserved.

Keywords: Self-assembled monolayers; Adsorption; Molecular simulation; Gold nanocrystals

\section{Introduction}

The engineering of materials on the nanometer scale is generating much excitement in modern electronics, optics, catalysis, ceramics, magnetic storage, and biophysics. Important classes of nanomaterials with special size- and shape-dependent optical and electrical functionality include gold- and semiconductor crystallites of size $2-10 \mathrm{~nm}$. These crystallites are called nanocrystals (NCs) and can assemble in two- and three-dimensional superlattices.

NCs are often protected by an organic capping layer that prevents aggregation, e.g., gold NCs are often capped with alkyl thiol molecules. While self-assembled monolayers of alkyl thiols have been studied extensively, less is known about the structure of these molecules on gold NCs. Experiments show that temperature-induced phase transitions of the capping layer can dramatically change optical properties of NCs in solution [1]. This is due to the reconstruction of the NC surface which is induced by the capping layer. Therefore, it is of considerable importance to study how the internal structure of molecules in the capping layer depends on the geometry (size, shape and curvature) of NCs.

In this preliminary study, we focus on the differences between adsorption of alkyl thiols on a flat gold surface and adsorption on the facets of a gold NC. It turns out that small

\footnotetext{
* Corresponding author.

E-mail address: t.j.h.vlugt@phys.uu.nl (T.J.H. Vlugt).
}

NCs significantly disturb the optimal structure of the alkyl thiol molecules.

\section{Simulation methodology}

\subsection{Model}

The systems we study contain either a single icosahedral gold NC or a close-packed gold (111) slab, which are kept rigid, and unbranched alkyl thiol surfactants, with $n$-hexane as a solvent. To model the nonbonded interactions, we use a united atom model for the surfactant and solvent molecules.

In the united atom approach, the $\mathrm{CH}_{x}(x=2,3)$ and $\mathrm{SH}$ segments are represented by single $\mathrm{C}$ and $\mathrm{S}$ pseudo-atoms, respectively. The intermolecular interactions are described by a Lennard-Jones (LJ) potential which is truncated and shifted at $12.0 \AA$. Our LJ parameters are summarized in Table 1. We include intramolecular bond stretching, bond bending, a torsional potential and LJ interactions between united atoms separated by more than three bonds [2]. The united atoms of the solvent and surfactant molecules interact with Au atoms via LJ interactions as well. The choice of the potential parameters (Table 1) for the $\mathrm{Au}-\mathrm{S}$ and $\mathrm{Au}-\mathrm{CH}_{x}$ interactions are justified in the Section 3.1.

\subsection{Monte Carlo simulations}

All simulations are performed in the grand-canonical $(\mu \mathrm{VT})$ ensemble at $T=300 \mathrm{~K}$. The Configurational-Bias Monte Carlo 
Table 1

LJ parameters used in this study

\begin{tabular}{lrrrr}
\hline$\epsilon_{i j} / k_{B}$ & $\mathrm{CH}_{3}$ & $\mathrm{CH}_{2}$ & \multicolumn{1}{l}{$\mathrm{S}$} & \multicolumn{1}{c}{$\mathrm{Au}$} \\
{$[\mathrm{K}]$} & & & & \\
\hline $\mathrm{CH}_{3}$ & 108 & 78 & 117 & 108 \\
$\mathrm{CH}_{2}$ & 78 & 56 & 84 & 88 \\
$\mathrm{~S}$ & 117 & 84 & 126 & 4260 \\
$\mathrm{Au}$ & 108 & 88 & 4260 & - \\
\hline
\end{tabular}

\begin{tabular}{lllll}
\hline$\sigma_{i j}[\AA]$ & $\mathrm{CH}_{3}$ & $\mathrm{CH}_{2}$ & $\mathrm{~S}$ & $\mathrm{Au}$ \\
\hline $\mathrm{CH}_{3}$ & 3.76 & 3.86 & 4.11 & 3.54 \\
$\mathrm{CH}_{2}$ & 3.86 & 3.96 & 4.21 & 3.54 \\
$\mathrm{~S}$ & 4.11 & 4.21 & 4.45 & 2.40 \\
$\mathrm{Au}$ & 3.54 & 3.54 & 2.40 & - \\
\hline
\end{tabular}

Values for $\epsilon_{\mathrm{CH}_{x}-\mathrm{S}}$ and $\sigma_{\mathrm{CH}_{x}-\mathrm{S}}$ are obtained by applying the mixture rules $\epsilon_{i j}=\sqrt{\epsilon_{i i} \epsilon_{j j}}$ and $\sigma_{i j}=\left(\sigma_{i i}+\sigma_{j j}\right) / 2$, respectively. $\mathrm{CH}_{x}-\mathrm{CH}_{x}$ parameters were taken from Ref. [2]. The S-S interaction parameters were taken from Refs. $[3,4]$.

(CBMC) [5] scheme has become the method of choice for the simulation of medium-length chain molecules. The following trial-moves are used: (1) Translation of a surfactant or solvent molecule. (2) Rotation of a surfactant or solvent molecule. (3) Partial or complete regrow of a surfactant or solvent molecule using the CBMC algorithm. In case of a complete regrow multiple trial positions for the first bead are used. (4) Insertion or removal of solvent molecules using the CBMC algorithm. The chemical potential of the solvent equals the chemical potential of a pure solvent at the same temperature. (5) Identity changes for surfactant molecules using the CBMC algorithm [6]. The acceptance rules of these trial moves can be found in Refs. [5,7].

The molfraction of each surfactant type is controlled by their difference in chemical potential. This quantity has been converted into the ratio of their molfractions in a bulk solvent (without a gold structure present) at the same chemical potential difference. This requires an additional simulation that is performed in the Henry regime, i.e. the total number of surfactant molecules is very small compared to the number of solvent molecules.

One of the possible difficulties in our MC simulations is to ensure that the system is well equilibrated. During a MC simulation on a rigid gold surface, a alkyl thiol molecule adsorbed with its SH-group in the gap between three gold atoms will most likely stay there due to the extremely strong $\mathrm{Au}-\mathrm{S}$ interaction. Therefore, configurations where all thiol groups are adsorbed like this are very stable and may not change significantly during long simulations. In contrast to this, experiments suggest a hexagonal overlayer of thiols with S-S spacing of $4.97 \AA$ [8]. To circumvent any equilibration problems, our starting configurations are equilibrated using parallel tempering with respect to the $\mathrm{Au}-\mathrm{S}$ interaction strength [9]. The radial distribution function of the thiol groups $g_{\mathrm{SS}}(r)$ (Fig. 1) indeed shows that we have such a realistic sulfur arrangement on the surface. For a $\mathrm{NC}$, there are more possible adsorption sites, leading to a reduced S-S spacing (Fig. 1). Furthermore, on the NC, there is less structure in the arrangement of adsorbed thiol groups.

\section{Results and discussion}

\section{1. $A u-C$ and $A u-S$ force field development}

In order to make a direct comparison between the adsorption behavior of alkyl thiols on a gold (111) surface and a gold

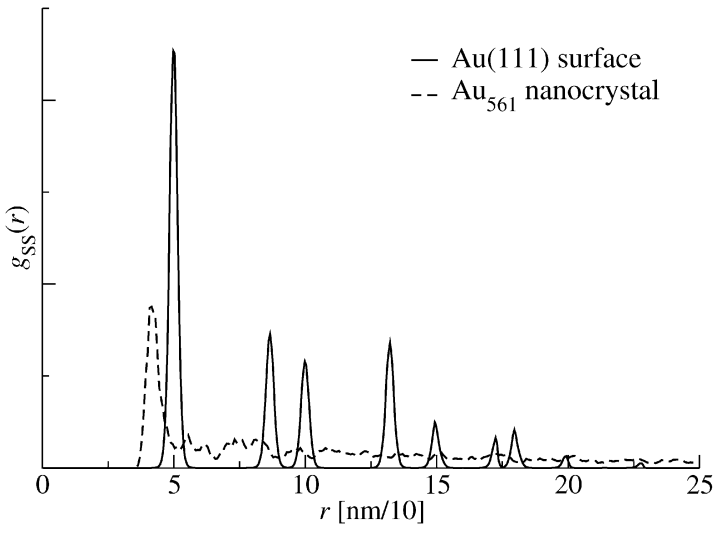

Fig. 1. Radial distribution function $g_{\mathrm{SS}}(r)$ for a gold (111) surface and a $\mathrm{Au}_{561}$ $\mathrm{NC}\left(96 / 124\right.$ adsorbed $\mathrm{CH}_{3}\left(\mathrm{CH}_{2}\right)_{4} \mathrm{SH}$ molecules, respectively).

Table 2

Comparing $V^{\text {tot }}\left(z^{\prime}\right)$ and $V^{\text {eff }}(z)$ by Hautman and Klein [10]

\begin{tabular}{lllll}
\hline Species & $z_{m}[\AA]$ & $z_{m}^{\prime}[\AA]$ & $V^{\text {eff }}\left(z_{m}\right) / k_{B}[\mathrm{~K}]$ & $V^{\text {tot }}\left(z_{m}^{\prime}\right) / k_{B}[\mathrm{~K}]$ \\
\hline $\mathrm{CH}_{2}$ & 3.51 & 3.52 & -683 & -678 \\
$\mathrm{CH}_{3}$ & 3.51 & 3.48 & -832 & -831 \\
$\mathrm{~S}$ & 2.40 & 2.40 & $-1.4 \times 10^{4}$ & $-1.4 \times 10^{4}$ \\
\hline
\end{tabular}

$\mathrm{NC}$, we fitted effective Lennard-Jones parameters for the Au$\mathrm{CH}_{x}$ and $\mathrm{Au}-\mathrm{S}$ interactions based on the well-established and widely-used effective potential of Hautman and Klein [10] for $\mathrm{Au}(111)$. This potential is of the form

$V^{\text {eff }}(z)=\frac{C_{12}}{\left(z-z_{0}\right)^{12}}-\frac{C_{3}}{\left(z-z_{0}\right)^{3}}$,

where $z$ is the distance from the surface and $z_{0}, C_{12}$ and $C_{3}$ are constants which determine the position $z_{m}$ at the potential minimum $V^{\text {eff }}\left(z_{m}\right)$. We constructed a rectangular gold fcc slab (lattice spacing $4.085 \AA$ ) of dimension $32 \times 32 \times 30$ with the (111) surface facing a single $\mathrm{C}$ (or S) segment at a given distance $z^{\prime}$ from that surface. Next, we computed the total interaction energy $V^{\text {tot }}\left(z^{\prime}\right)$ as follows. For 300 separations $z^{\prime}$ of range $2 \AA<$ $z^{\prime}<15 \AA$, we randomly generated 1000 coordinates on a circle of radius $r=5 \AA$ and determined the sums of all pair interactions between the segment and the gold slab. We took $V^{\text {tot }}\left(z^{\prime}\right)$ as the average total energy for each $z^{\prime}$. The LJ parameters (Table 1) were adjusted such that the minimum value of $V^{\text {tot }}$ as well as the position of the minimum $z^{\prime}=z_{m}$ corresponded to the effective potential $V^{\text {eff }}\left(z^{\prime}\right)$ from Ref. [10], see Table 2 . Additionally, the shapes of our calculated effective potentials closely resemble the ones by Hautman and Klein [10]. Furthermore, the $\mathrm{LJ}$ potential well depth $\epsilon_{\mathrm{S}-\mathrm{Au}}$ is almost identical to the potential well depth used by Landman and co-workers [3].

\subsection{Mixtures of alkyl thiols}

To investigate the composition of co-adsorbed alkyl thiols, we carried out simulations of binary thiol mixtures adsorbed on gold in presence of the solvent ( $n$-hexane) for both a (111) slab and a NC. We also performed the same type of simulations in systems without a gold structure to enable comparison between bulk solution and surface composition. In this paper, we discuss 

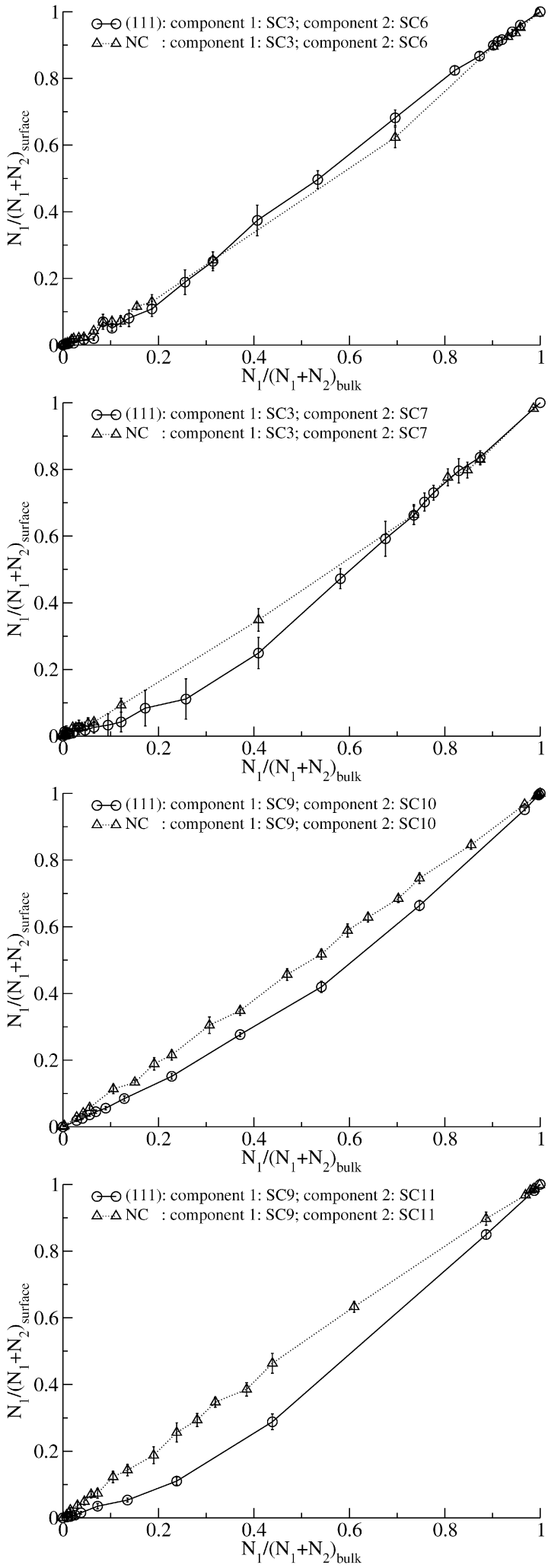

Fig. 2. Adsorption selectivities for SC3-SC6, SC3-SC7, SC9-SC10 and SC9-SC11.

the following mixtures: SC3-SC6; SC3-SC7; SC9-SC10 and SC9-SC11.

The box size for the simulations with the gold slab is $34.620 \AA \times 29.982 \AA \times 100 \AA$, periodic boundary conditions
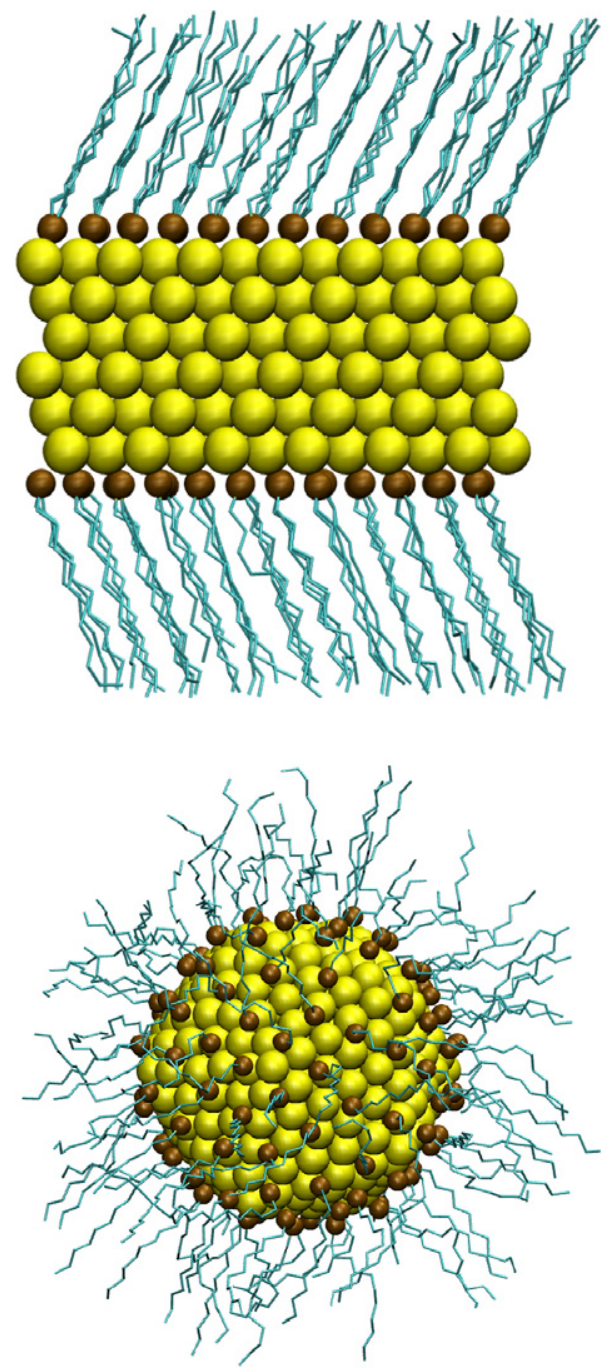

Fig. 3. Typical snapshots for adsorption of a SC9-SC11 mixture on Au(111) and a gold NC. The solvent molecules are not shown.

are applied. The gold slab consists of $12 \times 12 \times 6$ atoms and has two adsorbing surfaces. In the initial configuration, the surface is fully covered with alkyl thiols (here: 96) which are arranged in a hexagonal overlayer, just as for a simulation using the effective interactions of Eq. (1). For the simulations including a $\mathrm{Au}_{561} \mathrm{NC}$, we used a cubic simulation box of dimension $60 \AA$, containing 124 adsorbed alkyl thiols and applied similar simulation conditions as in the gold slab simulations.

The results are presented in Fig. 2. The adsorption selectivity for NCs is almost identical to that in the pure solvent. This is quite different for $\mathrm{Au}(111)$, where the longest alkyl thiol is preferentially adsorbed [11]. This is due to differences in interaction strength between the surfactants themselves, see Fig. 3 . $\mathrm{On} \mathrm{Au}(111)$ the surfactants are ordered and adopt a tilted configuration. On a NC however, this tilted structure is frustrated and destroyed by the icosahedral structure of the NC. This frustration will become less for larger NCs. As the facets of the icosahedral NC also have an (111) structure, we expect that this frustration will disappear eventually. 


\section{Conclusions}

In summary, we studied the adsorption of alkyl thiol surfactants on the $\mathrm{Au}(111)$ surface as well as on gold NCs. The adsorption selectivity on gold NCs is almost comparable to that in pure hexane, but this is different for a (111) surface.

\section{Acknowledgements}

T.J.H.V. acknowledges financial support from the Netherlands Organization for Scientific Research (NWO-CW) through a VIDI grant.

\section{References}

[1] S.F. Wuister, A. van Houselt, C. de Mello Donegá, D. Vanmaekelbergh, A. Meijerink, Angew. Chem. Int. Ed. 43 (2004) 3029.

[2] D. Dubbeldam, S. Calero, T.J.H. Vlugt, R. Krishna, T.L.M. Maesen, B. Smit, J. Phys. Chem. B 108 (2004) 12301.

[3] W.D. Luedtke, U. Landman, J. Phys. Chem. B 102 (1998) 6566.

[4] M. Lal, M. Plummer, N.J. Richmond, W. Smith, J. Phys. Chem. B 108 (2004) 6052.

[5] D. Frenkel, B. Smit, Understanding Molecular Simulations, second ed., Academic Press, San Diego, USA, 2002.

[6] M.G. Martin, J.I. Siepmann, J. Am. Chem. Soc. 119 (1997) 8921.

[7] M. Macedonia, E. Maginn, Mol. Phys. 96 (1999) 1375.

[8] C. Widrig, C. Alves, M. Porter, J. Am. Chem. Soc. 113 (1991) 2805.

[9] T.J.H. Vlugt, B. Dünweg, J. Chem. Phys. 115 (2001) 8731.

[10] J. Hautman, M. Klein, J. Chem. Phys. 91 (1989) 4994.

[11] A.V. Shevade, J. Zhou, M.T. Zin, S. Jiang, Langmuir 17 (2001) 7566. 